Classification

Physics Abstracts

$71.20-\mathrm{b}-71.25 \mathrm{Mg}-79.20 \mathrm{Hx}$

\title{
Electron energy and momentum spectroscopy in solids
}

\author{
Kaare J. Nygaard, Yu Chen $\left(^{*}\right)$, Julian Lower, Peter Storer and Erich Weigold
}

Electronic Structure of Materials Centre, The Flinders University of South Australia, Bedford Park, S.A. 5042, Australia

(Received October 11, 1990; accepted January 28, 1991)

\begin{abstract}
We have used (e,2e) coincidence techniques to study electron momentum densities as a function of binding energy and momentum in thin films. The experiment employs symmetric coplanar geometry with incident electron beam energy of $10 \mathrm{keV}$. The outgoing electrons are retarded to about $100 \mathrm{eV}$ before being dispersed in identical hemispherical analysers and counted in one-dimensional position-sensitive detectors consisting of multichannel plates and resistive anodes. Binding energy spectra are obtained by stepping the incident energy through a predetermined range. In amorphous carbon films $80 \AA$ thick, we have obtained electron momentum distributions for binding energies from 0 to $40 \mathrm{eV}$ with momentum as parameter $(0,0.4$ and 0.8 atomic units). The results clearly show contributions from the $\pi$ - and $\sigma$ - bands and demonstrate the usefulness of the method.
\end{abstract}

\section{Introduction.}

The basic principle of EELS is to excite bound-bound transitions in atoms by high-energy electrons from which energy-filtered images and local energy loss spectra can be obtained. An energy loss resolution of $1 \mathrm{eV}$ is routinely achieved in commercially available instruments as reported by Krivanek [1], while the retardation method employed by Batson [2] has a resolution of about $0.15 \mathrm{eV}$. The collection of papers included in this issue is mostly devoted to EELS technology, analysis and elemental sensitivity. The one parameter which is not addressed by the EELS community is the momentum $\mathbf{q}$ of electrons occupying a certain band $i$ with energy $\epsilon_{i}(\mathbf{q})$.

The only method capable of providing direct information on the probability density of finding an electron in a band with energy $\epsilon_{i}$ and momentum $\mathbf{q}$ for a large range of $\mathbf{q}$-values is based on the $(e, 2 e)$ coincidence method. Complete knowledge of energies and momenta of incident and exit electrons provides information on binding energies $\epsilon_{i}$ and momenta $q$ of the bound electrons. (The binding (or separation) energy $\epsilon_{i}$ is defined as the energy needed to move a bound electron $\left(\epsilon_{i}, \mathbf{q}\right)$ to infinity.) In atomic and molecular physics the method is commonly known as electron momentum spectroscopy (EMS). Here we shall use this method to investigate phenomena in solids.

Other methods which have been employed to deduce information on momentum distributions and energy-momentum dispersion curves include Compton scattering and photoelectron spec-

$\left(^{*}\right)$ Present address: 2312 Silver Lane (Apt. 301), New Brighton, MN 55112, U.S.A. 
troscopy. It should be noted that Compton profiles, which are integrated momentum densities summed over all occupied states, give momentum densities which are intrinsically inaccurate for small $q$-values and give a probability density averaged over all occupied valence plus core states. Angle-resolved photoelectron spectroscopy (ARPES) is a good tool for obtaining dispersion curves, but it cannot provide momentum density information.

The object of this report is to describe an electron momentum spectrometer specially designed for the study of valence electrons in solids and to present the first high energy resolution results in carbon.

The material in this paper is organized as follows: In section 2 we give a theoretical background of the (e,2e)-method as it applies to solids. The apparatus, which uses hemispherical analysers and position sensitive detectors, is described in section 3. The automated data acquisition system is presented in section 4, followed by typical results in amorphous carbon in section 5 .

\section{The (e,2e)-method.}

The notation (e,2e) symbolizes an ionization process caused by a fast incident electron followed by two outgoing electrons leaving an ionized target behind. Since the energies and momenta of all "external" electrons are known, the salient features of the method are that both electron binding energies and momenta of bound electrons can be accurately determined. Recent reviews of EMS have been made by McCarthy and Weigold [3] and by Weigold [4]. The methodologies developed in atomic and molecular physics at the Flinders University of South Australia have led to the development of a high resolution electron momentum spectrometer specially designed for thin film investigations.

The problem at hand is to determine the probability density of finding an electron in a solid with momentum $\mathbf{q}$ and binding energy $\epsilon_{i}$. This is accomplished by the clean knockout of a bound electron from the target and measuring its energy and momentum. If the energies and momenta of the incident and exit electrons are defined to be $E_{0}, E_{\mathrm{A}}, E_{\mathrm{B}}$ and $\mathbf{p}_{0}, \mathbf{p}_{\mathrm{A}}, \mathbf{p}_{\mathrm{B}}$, respectively, the system is kinematically described by the equations for conservation of energy and momentum by

$$
E_{0}=E_{\mathrm{A}}+E_{\mathrm{B}}-\epsilon_{i}(\mathbf{q})
$$

and

$$
\mathbf{p}_{0}+\mathbf{q}=\mathbf{p}_{\mathrm{A}}+\mathbf{p}_{\mathrm{B}}
$$

The equations describing conservation of energy and momentum are pictorially illustrated in figure 1 , which, before the collisional events, depicts a bound electron (filled circle) in momentum space being bombarded by a fast electron $\left(\mathbf{p}_{0}, E_{0}\right)$. After the collision, two electrons $\left(\mathbf{p}_{\mathbf{A}}, E_{\mathrm{A}}\right)$ and $\left(\mathbf{p}_{\mathrm{B}}, E_{\mathrm{B}}\right)$ move away from the target area, leaving a positive hole (open circle) behind.

In practice, the two outgoing electrons are detected in coincidence to ensure that they originate from the same specific event. If $p_{\mathrm{A}} \geq p_{\mathrm{B}}$ it is customary to refer to electron $\mathrm{A}$ as the "scattered electron". The momentum transferred by the scattered electron to the target is

$$
\mathbf{K}=\mathbf{p}_{0}-\mathbf{p}_{\mathbf{A}} \text {. }
$$

We note that high momentum transfer implies close electron-electron collisions. Thus, for high incident energies $\left(E_{0} \gg \epsilon_{i}\right)$ and sufficiently high momentum transfer $\mathbf{K}$, the target electron is cleanly knocked-out, leaving a positive hole behind. The momentum transfer is maximized for $E_{\mathrm{A}}=E_{\mathrm{B}}$ and for polar angles $\theta_{\mathrm{A}}=\theta_{\mathrm{B}}=\theta$. This socalled symmetric kinematics scheme is further simplified by setting the azimuthal angle $\phi=0$, that is, the incident and exit electron trajectories 

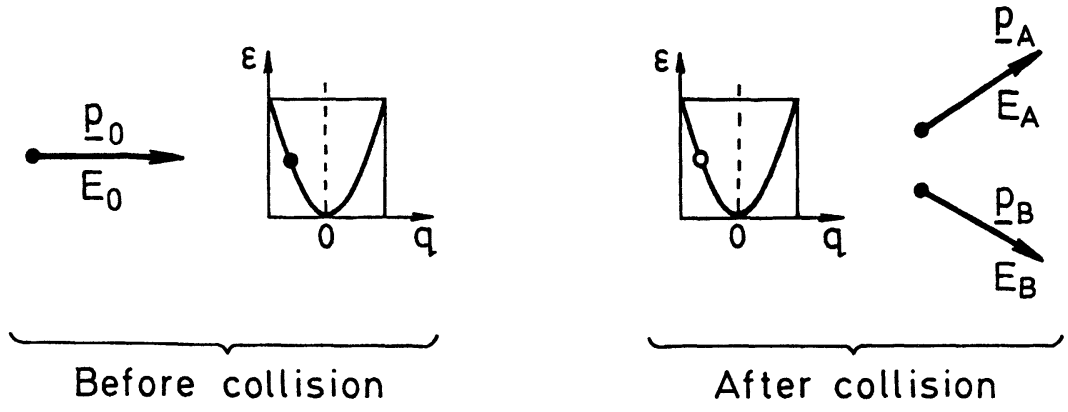

Fig. 1. - Principle of (e,2e) experiment.

all lie in the same plane. Unfortunately in this arrangement target momenta are fixed for fixed $\theta$ and restricted to zero for $\theta=45^{\circ}$. However, by varying the energy sharing between $E_{\mathrm{A}}$ and $E_{\mathrm{B}}$, i.e. setting

$$
\begin{aligned}
& E_{\mathrm{A}}=\frac{1}{2}\left(E_{0}+\epsilon_{i}\right)+\Delta E \\
& E_{\mathrm{B}}=\frac{1}{2}\left(E_{0}+\epsilon_{i}\right)-\Delta E
\end{aligned}
$$

thus keeping $\left(E_{\mathrm{A}}+E_{\mathrm{B}}\right)$ constant, it is possible to probe different values of $\mathbf{q}$. A simple calculation using equations (2) and (4) yields

$$
q=p_{0} \frac{\Delta E}{E_{0}}\left(1+\frac{\epsilon_{i}}{E_{0}}\right)
$$

Vector analysis shows that the target momentum direction available for study in the energy sharing method is in the collision plane and perpendicular to the incident electron beam (McCarthy and Weigold [5]). The advantage of the symmetric coplanar geometry is that a wide range of $q$ values can be studied by floating the two analysers at different voltages $V \pm \Delta V$. An additional advantage is that there are no moving parts. In the experiment, for $E_{0}=10 \mathrm{keV}$ and $\epsilon_{i} \approx 20 \mathrm{eV}$, the binding energy correction to $q$ in equation (5) is only of the order of $0.2 \%$.

\section{Apparatus.}

A schematic diagram of the symmetric coplanar apparatus is shown in figure 2. Electrons of incident energy $E_{0}$ impinge upon a thin film. The two outgoing electrons are energy analyzed in identical hemispherical analysers $A$ and $B$ detected in coincidence. The apparatus is pumped out to a pressure of a few nano Torr by a turbomolecular pump. The earth's magnetic field is cancelled out to within $\pm 2 \mathrm{~m}$ Gauss by Helmholz coils.

Further details of the electron trajectory part of the apparatus are shown in the lower left portion of figure 3. The primary electron beam is generated in a commercial electron gun (Cliftronics Model $83 \mathrm{CE}$ ) which produces a $0.5 \mathrm{~mm}$ diameter, $5 \mu \mathrm{A}, 10 \mathrm{keV}$ beam with an energy spread of about $0.7 \mathrm{eV}$. The focussing properties of the beam are monitored in a segmented Faraday cup/beam dump positioned behing the interaction region. The interaction region is defined by the intersection of the beam and the target consisting of a free-standing $80 \AA$ thick amorphous 


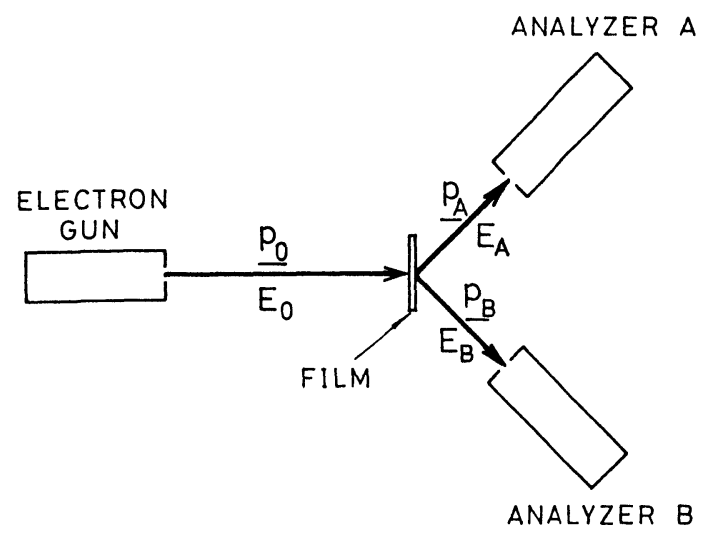

Fig. 2. - Schematic diagram of the symmetric coplanar apparatus. The two analysers are floating at different potentials relative to ground to establish desired momentum values. The target electron momentum $\mathbf{q}$ is in the collision plane and perpendicular to $\mathbf{p}_{0}$.

carbon film (Arizona Carbon Foil Co., Inc.) mounted over a $2 \mathrm{~mm}$ diameter aperture. Scattered electrons leaving the target at angles of $45^{\circ}$ relative to the incident beam direction are transported through two identical electrostatic hemispherical analysers positioned on opposite sides of the beam. Each analyser is preceded by a 5-element electrostatic retarding and focussing lens system which defines the angular acceptance and hence momentum resolution ( 0.05 a.u.) and following deceleration, focusses the beam of electrons onto the entrance plane of the analyser. Each analyser has an energy range of $20 \mathrm{eV}$ centred about a mean pass energy of $100 \mathrm{eV}$.

In each analyser, the arrival time and energy of the electrons are determined in the exit plane by a position-sensitive detector consisting of dual microchannel plates and a resistive anode. To a good approximation, the arrival position of an electron is proportional to its energy as discussed by Lower and Weigold [6]. The energy resolution for the combination of the electron beam (at $5 \mathrm{keV}$ energy), lenses, hemispherical analyser, and position sensitive detector was measured using elastic scattering from the film at $45^{\circ}$ and amounts to $1 \mathrm{eV}$. The energy $v$ s exit position is linear to within $\pm 2.5 \%$ over the energy window of $20 \mathrm{eV}$. The total energy resolution for the coincidence measurements is estimated to be $1.5 \mathrm{eV}$.

\section{Data acquisition.}

The purpose of the electronics scheme illustrated in figure 3 is to distinguish pairs of electrons originating from specific ionization events by their arrival times and energies at the two detectors. As part of the process, background counts due to noncorrelated events need to be subtracted from the total counts. Making reference to the "timing" spectrum shown in figure 4, we define $N_{\mathrm{c}}$ to be the number of counts recorded in the coincidence window of width $\Delta t_{\mathrm{c}}$. In order to improve on the statistical accuracy, the background counts $N_{\mathrm{b}}$ are accumulated in a wider region $\Delta t_{\mathrm{b}}$, where $\Delta t_{\mathrm{b}}=R \Delta t_{\mathrm{c}}$. The true coincidence counts accumulated over a time $T$ is then simply given by

$$
N_{\mathrm{t}}=N_{\mathrm{c}}-\frac{N_{\mathrm{b}}}{R}
$$

Typically, in the experiment, the ratio of true coincidence counts to background counts within the coincidence window is 1:1. Further improvement in this "signal-to-noise" ratio are difficult 


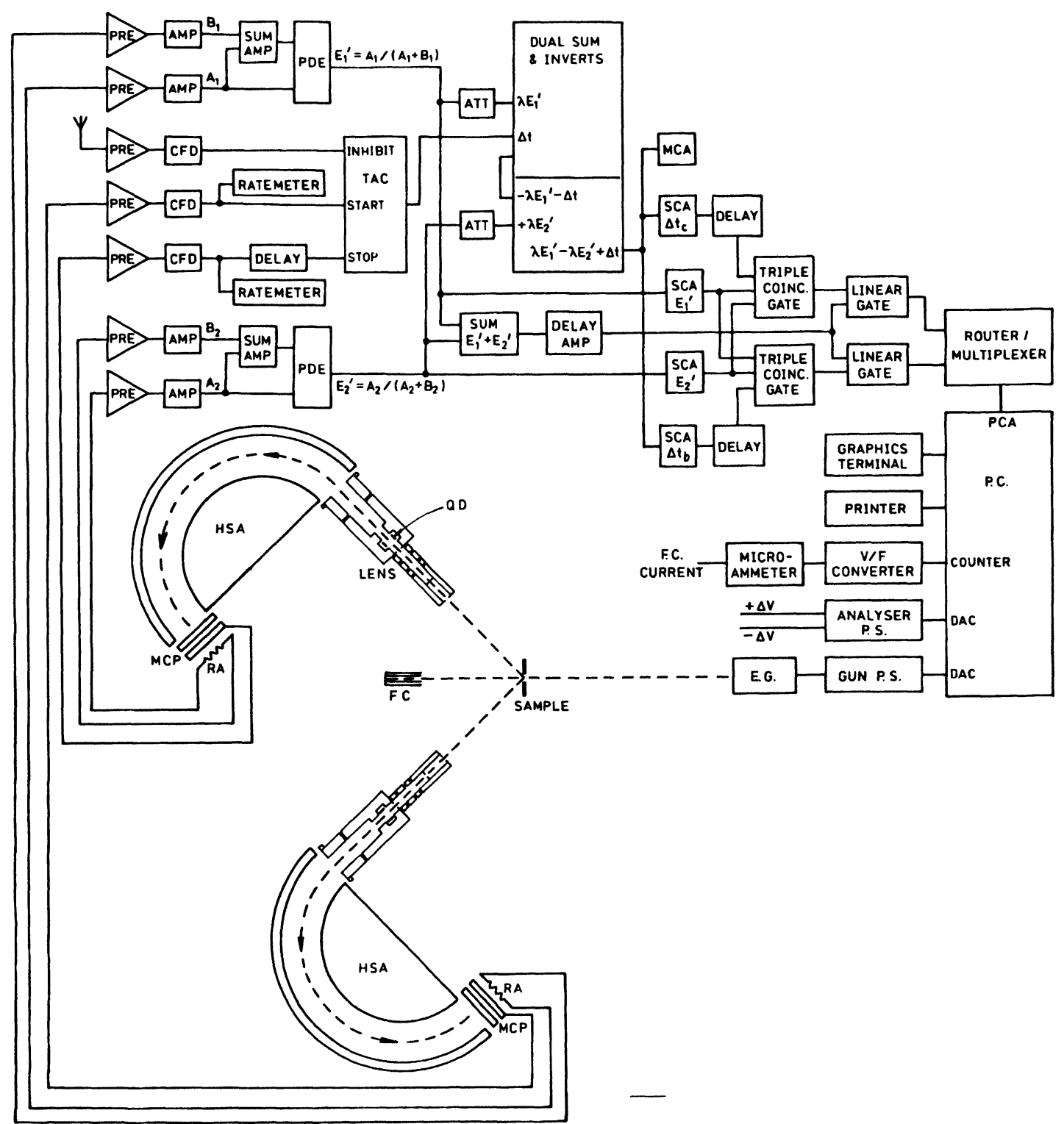

Fig. 3. - Schematic diagram of the apparatus and associated circuit which distinguishes pairs of electrons originating from single collision events by their relative arrival times at two detectors and determines the momentum and energy of each electron in a pair. The circuit makes correction for the energy-dependent time of flight variations which occur within the analysers. $A_{1}, B_{1}, A_{2}, B_{2}$ pulse amplitudes; ADC analogue to digital converter; ATT attenuator; CFD constant fraction discriminator; EG electron gun; FC Faraday cup; HSA hemispherical analyser; MCP microchannel plates; PDE position decoding electronics; PRE preamplifier; RA resistive anode; SCA's single channel analysers for signal, background and energy windows; TAC time to amplitude converter.

to accomplish since $N_{\mathrm{t}}$ is proportional to the beam current and $N_{\mathbf{b}}$ is proportional to the current squared. Further details are discussed by Lower and Weigold [6].

The coincidence technique used in this experiment employs both fast and slow coincidence 


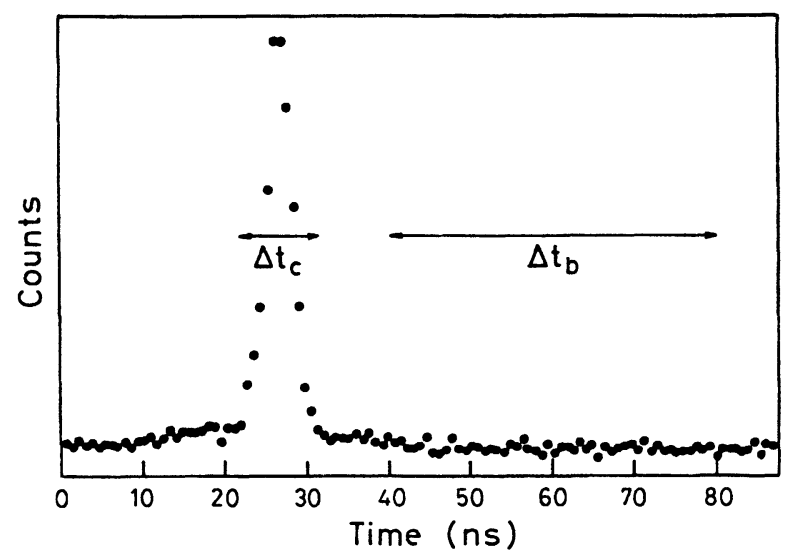

Fig. 4. - Timing spectrum showing the distribution of relative arrival times between detected electrons. Pairs of electrons derived from single collisions form a peak of coincident events and are measured in the signal window $\Delta t_{c}$. Events due to the detection of pairs of electrons from different collisions are collected in the background window $\Delta t_{\mathrm{b}}$. The F.W.H.M. of the coincidence peak is $4.5 \mathrm{nsec}$.

channels. The fast pulses determining electron times of arrival are obtained inductively from back of the microchannel plates. The slow pulses are capacitively coupled from the two ends of the resistive anodes and used for energy analysis in the position sensitive electronics.

In the fast channel, timing pulses from the fast preamplifiers enter constant fraction discriminators, which set threshold levels to remove noise from the signal lines and produce an output pulse correlated in time to each input signal. The discriminator output from one detector is used to start a time-to-amplitude converter (TAC) and the delayed output from the other detector provides the stop pulses. The TAC produces an output pulse proportional in height to the measured time differences between the start and stop input pulses. A noise antenna is connected to an identical third preamplifier-discriminator network and generates a veto pulse to inhibit data collection in the TAC upon detection of fast noise.

In the slow channels, the energies of the exit electrons in each coincidence event are determined. Pulses from the two ends of the resistive anode are amplified and summed. The summed pulse amplitude is proportional to the total charge deposited on the anode. Furthermore, the amplitude of the pulse from one end of the anode, when divided by the amplitude of the summed pulse, gives a value which varies linearly with the arrival position of an electron at the analyser exit plane. The analogue division is performed through position decoding electronics (PDE) developed by ORTEC. Corrections for the effects of energy-dependent time of flight variations within the analysers are made in the following manner [6]: In the first sum and invert amplifier shown in the top middle part of figure 3 the position signal $E_{1}^{\prime}$ is attenuated by a factor $\lambda$ and combined with the corresponding output pulse $V(\Delta t)$ from the TAC. The position signal $E_{2}^{\prime}$ from the second detector is attenuated by the same factor and added to the output from the first inverted amplifier. The final inversion provides an output pulse proportional to $\left[V(\Delta t)+\lambda\left(E_{1}^{\prime}-E_{2}^{\prime}\right)\right]$, which exhibits a minimum width for $E_{1}^{\prime}=E_{2}^{\prime}$.

When displayed upon a multichannel analyser (MCA), the corrected TAC output pulses generate the "timing spectrum" shown in figure 4. This spectrum shows a peak of "true" coincidence events, resulting from pairs of electrons derived from single collision events, superimposed upon a flat background of "accidental events" resulting from electron pairs in which the two contributing electrons are derived from different collision events. To identify the "true" and "accidental" 
coincidence events, the corrected timing pulses are fed into two single channel analysers (SCA). One, the signal SCA, has its window $\Delta t_{\mathrm{c}}$ centred around the "true" coincident peak whilst the second, referred to as the background SCA, measures a wider region $\Delta t_{\mathrm{b}}$ of the timing spectrum far away from the peak.

Having identified events corresponding to true and accidental coincidences in the fast channel, two triple coincidence gates (TCG's) are used to ensure that each TAC output pulse accepted through either SCA window is accompanied by two valid position output pulses, one from each analyser. For this purpose, two additional SCA's are used to observe the PDE output pulses from each analyser and generate logic pulse inputs to the TCG's. These SCA's additionally allow the range of accepted energies from each analyser to be controlled electronically, should this be required. Subsequently, the outputs from the two TCG's are used to gate two linear gates, whose input pulses are derived from the summed PDE output pulses from the two analysers. The two linear gate outputs are then fed into a multiplexer and analogue-to-digital converter, the output of which is accumulated in the computer. Thus, two coincidence energy spectra are collected, one corresponding to events detected in the signal timing window, which includes the true coincidences, the other due to events detected in the background timing window, resulting purely from uncorrelated scattering events. The number of true coincidence events accumulated over a time $\mathrm{T}$ is then computed according to equation (6).

Finally, in order to obtain a constant detection sensitivity over the summed spectral image, the incident electron beam energy is incremented in small steps by the computer which automatically stores the net coincidence counts in the correctly adjusted binding energy interval. The computer also controls the voltage $\pm \Delta V$ which is used to define the momentum according to equation (5).

\section{Results and discussion.}

A representative selection of data deconvoluted to compensate for multiple scattering effects (Jones and Ritter [7]) is displayed in figure 5. The maximum which appears at $21 \mathrm{eV}$ for $q=0$ is attributed to ionization of electrons occupying the $\sigma_{1}$-band. This feature is strongly dispersed to an energy of about $16.5 \mathrm{eV}$ for $q=0.4 a_{0}^{-1}$, but has decreased in width in the $q=0.8 a_{0}^{-1}$ spectrum. The structure depicted below $11 \mathrm{eV}$ is attributed to the excitation of a graphitic $\pi$-band and shows dispersion of both the maximum and the low binding energy edge.

It is interesting to compare the data obtained here with those of Ritter, Dennison and Jones [8]. The most prominent band in their investigation of amorphous carbon appeared at about $23 \mathrm{eV}$ for $q=0$, exhibiting the same dispersive nature illustrated in figure 5. Similarly, they observed another band about $8 \mathrm{eV}$ below the Fermi energy which did not disperse as strongly as the $\sigma$-band. Since the energy resolution in their work was approximately $6 \mathrm{eV} \mathrm{F.W.H.M.,} \mathrm{the} \mathrm{details} \mathrm{in} \mathrm{their}$ spectra are not as pronounced as in figure 5.

The electronic structure of amorphous carbon is a topic still open for discussion. From measurements of electron Compton scattering, Williams, Sparrow, and Thomas [9] conclude that the bonding in amorphous carbon is more like graphite than diamond. However, as pointed out by Schattschneider and Pongratz [10] model calculations in graphite are too uncertain to warrant accurate prediction of Compton scattering data.

In our data it is noted that the momentum density for the lower-energy $\pi$-band at small $q$ values is significantly greater than expected from graphite calculations (Gao et al. [11]). Hence, the observed outer valence electron momentum densities indicate a non-graphitic bond.

It is of interest to compare the characteristics of the present equipment with those of other groups, as shown in table I. 


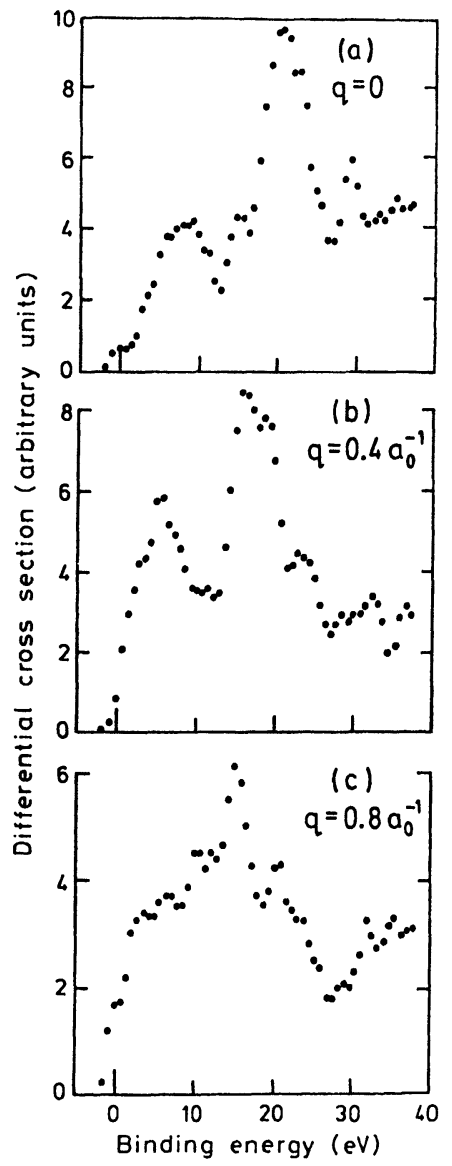

Fig. 5. - Deconvoluted coincidence binding energy spectra obtained at a series of target electron momenta $\left(0,0.4\right.$ and $\left.0.8 a_{0}^{-1}\right)$ from an $80 \AA$ amorphous carbon film. With increasing target electron momentum, the prominent peak structure of the $q=0$ spectrum ascribed to the ionization of the $\sigma_{1}$-band is seen to disperse towards the Fermi energy.

Most conspicuous in this table is the slow and remarkable improvement in the coincident energy resolution since 1969. Representative momentum resolution since 1988 are shown in the last column.

In order to improve on energy and momentum resolutions as well as data accumulation times we have developed a new spectrometer comprising one hemispherical and one toroidal analyzer, each equipped with a two-dimensional detector (see the bottom line in Tab. I). With the new instrument it will be possible to obtain coincidence data over a wide range of momentum-energy space in a few hours, as compared to 3 months in the experiment of Ritter et al. [8].

\section{Conclusions.}

We have constructed and tested an electron spectrometer for the study of momentum and binding energy spectra in thin films. The apparatus operates in the symmetric coplanar geometry in which the two outgoing electrons from an $(e, 2 e)$ reaction are counted in coincidence and energy 
Table I. - Historical development of electron momentum spectroscopy in solids.

\begin{tabular}{|l|c|c|c|c|l|}
\hline \multicolumn{1}{|c|}{ Group } & Anno & Ref. & $\begin{array}{c}E_{0} \\
(\mathrm{keV})\end{array}$ & $\begin{array}{c}\Delta E \\
(\mathrm{eV})\end{array}$ & $\begin{array}{c}\Delta q \\
\left(a_{0}^{-1}\right)\end{array}$ \\
\hline Amaldi et al. & 1969 & {$[12]$} & 14.7 & 135 & \\
Camilloni et al. & 1972 & {$[13]$} & 9 & 127 & \\
Krasil'nikova et al. & 1975 & {$[14]$} & 10 & 140 & \\
Persiantseva et al. & 1979 & {$[15]$} & 10 & 15 & \\
Ritter et al. & 1984 & {$[8]$} & 25 & 6 & \\
Dey and Williams & 1988 & {$[16]$} & 6 & 14 & \\
Gao et al. & 1988 & {$[11]$} & 25 & 6 & $0.25-0.4$ \\
Hayes et al. & 1988 & {$[17]$} & 7.5 & 4.5 & 0.6 \\
This work & 1990 & & 10 & 1.5 & 0.15 \\
New Spectrometer & 1991 & & 30 & 0.5 & 0.05 \\
\hline
\end{tabular}

analyzed by two identical hemispherical analysers. Each analyser is equipped with a positionsensitive electron detector comprising double microchannel plates and a resistive anode. Using a highly advanced data acquisition system which compensates for variations in detector sensitivities, a net coincidence count rate of a few counts per minute was achieved when a $5 \mu \mathrm{A}, 10 \mathrm{keV}$ electron beam bombarded an $80 \AA$ amorphous carbon film. The results in carbon demonstrate the feasibility of the method and reveals details not observed previously.

The use of dual two-dimensional detectors in our next generation spectrometers will reduce data accumulation time to a few hours.

\section{Acknowledgements.}

The authors would like to thank Dr. A. Beresford for software developments, Mr. R.S. Matthews for data analysis and Mrs. W. Inskip for typing the manuscript. The work reported here was sponsored by the Astralian Research Council.

\section{References}

[1] KRIVANEK O.L., Microsc. Microanal. Microstruct. 2 (1991) No2/3

[2] BATSON P.E., Ultramicroscopy 28 (1989) 32.

[3] MoCarthy I.E. and Weigold E., Rep. Prog. Phys. 51 (1988) 299.

[4] WEIGOLD E., J. Electron Spectrosc. Related Phenomena 51 (1990) 629.

[5] MCCARTHY I.E. and WEIGOLD E., Phys. Rep. 27C (1976) 275.

[6] LOWER J. and WeIGOLD E., J. Phys. E: Sci. Instrum. 22 (1989) 421.

[7] JONES R. and RITTER A.L., J. Electron Spectrosc. Related Phenomena 40 (1986) 285.

[8] RITTER A.L., DENNISON J.R. and JONES R., Phys. Rev. Lett. 53 (1984) 2054.

[9] Williams B.G., Sparrow T.G. and Thomas J.M., J. Chem. Soc., Chem. Commun. (1983), 1434.

[10] SCHATTSCHNEIDER P. and PONGRATZ P., Proceedings of 12th Werner Brandt International Conference on the Penetration of Charged Particles in Matter, September 4-7, 1989, San Sebastian, Basque Country, Spain, pp. 35-59. 
[11] Gao C., Ritter A.L., Dennison J.R. and HolzWARTH N.A.W., Phys. Rev. B 37 (1988) 3914.

[12] Amaldi Jr. U., Egidi A., Marconero R. and PizZelia G., Rev. Sci. Instrum. 40 (1969).

[13] CAMilloni R., Giardini-Guidoni A., Tiribelli R. and STEFANi G., Phys. Rev. Lett. 29 (1972) 618.

[14] Krasil'Nikova N.A., LeVIN V.G. and PERSIANTSEVA N.M., Sov. Phys. JETP 42 (1975) 796.

[15] Persiantseva N.M., Krasil'Nikova N.A. and Neudachin V.G., Sov. Phys. JETP 49 (1979) 530.

[16] Dey S. and Williams J.F., J. Phys. D: Appl. Phys. 21 (1988) 108.

[17] Hayes P., BenNetT M.A., FleXman J. and Williams J.F., Phys. Rev. B 38 (1988) 13371. 